\title{
Neuromuscular degeneration
}

\author{
After an angina attack, Mustafa Gunaydin was prescribed cholesterol lowering drugs, which seemed \\ to trigger neuromuscular degeneration, culminating in his death. His story is told by his partner
}

\author{
Caroline Fisher patient's partner ${ }^{1}$, Nicole Freris general practitioner ${ }^{2}$ \\ 'London N1 8DE, UK; ${ }^{2}$ Islington Central Medical Centre, London N1 1SW
}

\begin{abstract}
This is one of a series of occasional articles by patients about their experiences that offer lessons to doctors. The BMJ welcomes contributions to the series. Please contact Peter Lapsley

(plapsley@bmj.com) for guidance
\end{abstract}

\begin{abstract}
My partner, Mustafa, a long term smoker, underwent a single vessel angioplasty after an angina attack in September 2005, aged 55. He immediately stopped smoking and, on his doctor's advice, joined a three month rehabilitation exercise class and began taking statins ( $40 \mathrm{mg}$ simvastatin to start with, replaced by $40 \mathrm{mg}$ atorvastatin within two months). He continued to eat a healthy Mediterranean diet and take regular exercise (he was a semi-professional dancer in his youth and, having sold his business earlier in 2005 and taken early retirement, now enjoyed long walks exploring London with a friend). Although he had been a moderate drinker, in response to his health scare he cut down on alcohol. He was now free of angina, active, and positive, and said that he had never felt better.
\end{abstract}

\section{Early signs}

The first indications that all was not well came in spring 2006, when Mustafa started complaining of muscle cramps, especially at night, in his calves and thighs, usually after a long walk. By early 2007, he found it an effort to keep his arms aloft to wash his hair in the shower or to walk up even mild slopes. Sitting became uncomfortable, his neck and shoulders ached, and he experienced pain after mild exercise or even just standing for fairly short periods. He started to age visibly: his limbs lost muscle tone, his hair fell out, and he began to look permanently anxious as his confidence eroded. He had been proud of his looks and always dressed well, but now his clothes no longer fitted him and he had little spare energy to expend on his appearance.

He made several visits to his general practitioner over this period, and in August 2007 the statin dose was halved at his request. By October, his serum cholesterol level had risen to $5.4 \mathrm{mmol} / \mathrm{L}$, but, despite the lower statin dose, his creatine kinase level continued to rise and was now at 312 units/L.

\section{Things fall apart}

He became depressed about his permanent tiredness and weakness, and obsessed with finding out what was wrong with him, spending most days reading medical reference books. This fixation developed into a loss of trust with all but a few members of the medical profession with whom he came into contact during his illness. He felt that nobody was listening to him and that any discussion he wished to have about the documented side effects of statins and how they might be affecting him was met with a wall of silence. Two memorable remarks that contributed to his frustration were, "If you don't take the statins, you will die" (his GP) and "Ah, you're on statins, you will live forever" (a hepatologist).

\section{A welcome turnaround}

In April 2008 Mustafa decided to stop taking statins without telling his doctor. By late May, his symptoms had improved dramatically. He was able to walk long distances and up steep hills again, even in hot weather on a holiday in Turkey. The fact that his joie de vivre was returning was a huge boost for me too-it seemed that we could get our life back together again now that he was starting to have the energy to do the things he used to enjoy.

\section{Reversal of fortune}

At a check-up some time around the end of June, when his cholesterol level was found to have risen, he admitted that he had stopped taking statins. His GP strongly advised him to go back on them, and within a short time he was complaining of breathlessness, weakness, and backache. His GP switched him to ezetimibe $(10 \mathrm{mg})$, and within 10 days he was complaining of back and leg pain and a hoarse voice. His health continued to deteriorate, and by early November his voice was weak, he was fatigued all the time, and on one memorable night had to 
get up seven times to urinate. He developed a suffocating sensation when lying down, and his urine became brown and cloudy, but he was told that a urine test showed nothing abnormal. However, in late November his GP told him to stop taking ezetimibe immediately: we later discovered that his creatine kinase level had reached 783 units/L at that point.

Despite stopping all cholesterol lowering drugs, he continued to decline. By early 2009, his appetite had reduced, he had worsening muscle wasting, weakness, and twitching, especially at night, and in February was admitted to hospital. His emotional state at this time was poor; he was convinced he was going to die. While in hospital, he was seen by a neurologist whose notes state that there was "muscle twitching in thighs, which are rather coarse for true fasiculations" and who diagnosed statin induced myopathy. After a week, he was discharged from hospital without any real knowledge of what was likely to happen to him.

\section{Final months}

As the year progressed, he developed an itchy rash on the back of his legs, a dry mouth, mouth ulcers, and was often cold (particularly his legs, feet, and right shoulder), especially at night. He had vivid dreams, which he would act out; he was too weak to cough and could sleep only on one side, otherwise he couldn't breathe. During June, he had increased difficulty breathing and insisted on sitting in a chair all night, as he felt he would stop breathing if he went to sleep lying down. His posture was poor, and his neck became very weak. Eventually his head twisted round to the left, and his right shoulder was permanently raised. Several tests confirmed progressive weakness of his proximal muscles but normal strength in his limbs.

He had an appointment with a neurologist, who agreed with the statin related diagnosis and recommended a muscle biopsy if there was no improvement in his condition over the next three months. As his condition swiftly worsened, we got an emergency appointment at the same hospital, where he saw a different neurologist, who diagnosed, with little preamble and with nine people in the room, motor neurone disease. This came as a shock to us both and a huge blow to his already fragile mental state.

Despite this diagnosis, he had no muscle stiffness, no difficulty swallowing, and he never once fell. His fine dexterity was good: he was able to dress and feed himself until a few days before he died, only eventually needing help with these tasks because he was too weak to manage on his own. Two weeks before he died he threaded a needle, changed a fuse in a plug using a screwdriver, and fastened a small fiddly necklace clasp for me. I encouraged him to do as many of these tasks as possible in an effort to contradict a diagnosis that he found too awful to contemplate.

He died in hospital on 22 August 2009 of type 2 respiratory failure (oxygen level 50\% on admission to the emergency department) and two heart attacks over the course of three days.

\section{Listening to the patient}

During the last six months of his life, Mustafa came under the care of a different GP at the same practice. Dr Freris was kind and sympathetic and made appointments for him at times when I could be present too. She listened to his fears and concurred with his view that, whatever the final diagnosis, statins had had a serious effect on his health. Although it was clear to us all that he was beyond help, Mustafa always came away from those appointments feeling that he had found a doctor who understood what he was going through.

Many of the symptoms displayed during the course of Mustafa's illness - symptoms that eventually led to a diagnosis of motor neurone disease - are well documented side effects of statins and are listed in the leaflet that accompanies the tablets. With such similarities in mind, it seems wise to monitor statin-intolerant patients closely and to weigh up carefully whether the disadvantages of taking these drugs outweigh the benefits for these patients.

Competing interests: All authors declare: no support from any organisation for the submitted work; no financial relationships with any organisations that might have an interest in the submitted work in the previous three years, no other relationships or activities that could appear to have influenced the submitted work.

Provenance and peer review: Not commissioned; not externally peer reviewed.

Patient consent: Not needed.

Accepted: 25 April 2012

Cite this as: BMJ 2012;345:e6880

(c) BMJ Publishing Group Ltd 2012 


\section{A doctor's perspective}

I took over Mustafa's care in January 2009, when his health was in marked decline, and was impressed by his absolute conviction that statins had contributed to this deterioration. Understanding that cholesterol plays a vital role in cell health, particularly nerve cell function, and given the research that he presented, I took his concerns seriously. Supportive listening was probably the most important element of my care.

His inexorable decline without a clear cause was distressing and frustrating. As his GP, one of my roles was to hold an overview during his often difficult journey through the health system. He was referred to consultants from diverse specialties, each holding an important piece of the jigsaw-hepatologists (for his abnormal liver function test results), rheumatologists (for his myalgia), ENT consultants (for his hoarse voice), cardiologists (for his breathlessness), lipid clinics (for advice on statins), and neurologists (for his fatigue).

Mustafa's dying wish was that any connection between his final illness and statins be elucidated. GPs' actions are often driven by guidelines, treatment pathways, and quality and outcomes frameworks. Opportunity for careful consideration and contemplation of a disease process and manifestation is a rare luxury. Out of respect for Mustafa and concern for my profession's commitment to "do no harm," I have since spent much time researching the complex links between cholesterol, statins, and neurodegenerative disease.

Amyotrophic lateral sclerosis (ALS) is notoriously difficult to diagnose clinically, but certain aspects of Mustafa's presentation seemed inconsistent. Interestingly, symptoms were preceded by a disruption in liver function, which returned to normal on stopping statins. Mustafa presented with proximal myopathy developing one year after starting statins. Myalgia and fatigue remained the constant feature of his illness. His symptoms improved dramatically on stopping statins and became fulminant on restarting. His muscle strength was consistently assessed as normal, even as his death approached, although his proximal musculature and voice clearly showed fatigue.

Statins are among the most commonly used drugs, and motor neurone disease very rare, complicating confirmation of a causal link. There is, however, a growing body of evidence suggesting a connection. Raised serum cholesterol is protective in ALS and a marker for prognosis. Specialist centres now stop statins in new cases of ALS. There is evidence of an association between statins and the development of "ALS-like syndrome." What is not clear is whether statins unmask ALS or induce a similar degenerative process. One postulated mechanism of statin induced neuromuscular damage is blocking of coenzyme Q10 production, a key component of the mitochondrial respiratory chain and a powerful antioxidant. Reduced coenzyme Q10 levels are noted in several neurodegenerative disorders, including ALS. Although the precise mechanism is elusive, oxidative stress and mitochondrial dysfunction are now thought to be key influences in the development of ALS.

The inquiry precipitated by Mustafa's gentle insistence has supported my growing concern that vilification of cholesterol by the statin industry is distracting attention from the underlying cause for the epidemic of degenerative disease, including ALS, namely inflammatory and oxidatative stress resulting from our Western, grain based diet depleted of healthy fats and antioxidants.

Nicole Freris 